\title{
ASPECTOS DA BIOLOGIA DA REPRODUÇÃO E LONGEVIDADE DE COCHLIOMYIA MACELLARIA (FABRICIUS) (DIPTERA, CALLIPHORIDAE), EM CONDIÇÕES EXPERIMENTAIS. I. CASAIS AGRUPADOS
}

\author{
Sandra Lucia Cunha-e-Silva ${ }^{1}$ \\ Eliane M.V. Milward-de-Azevedo ${ }^{2}$
}

\begin{abstract}
BIOLOGICAL ASPECTS OF THE REPRODUCTION AND LONGEVITY OF COCHLIOMYIA maCellaria (FABricius) (Diptera, CAlliphoridaE) UNDER EXPERIMENTAL CONDITIONS. 1. GROUPED PAIRS. In the present study we estimed the longevity and reproductive potential of Cochliomyia macellaria (Fabricius, 1775), in different densities of grouped pairs, as a function of time of collection. The study was divided into two phases and conducted in an incubator regulated at $27^{\circ} \mathrm{C}, 65 \pm 10 \% \mathrm{RH}$ and 14 hours photoperiod. Forty and 20 pairs/replication were used, corresponding to the third and fourth generation originating from a laboratory colony. Egg masses were collected at 8:00 and 17:00 hours daily. The results obtained were influenced by the variables established. The ovipositing peak occurred 20 days after the emergence of females belonging to the third generation. This peak occurred earlier in fourth generation females, i.e., 11 days after imago emergence. These peaks corresponded to a total of 0,30 and $0,23 \mathrm{~g}$ of egg masses, respectively. The survival curves for adults were estimated by the Weibull method.

KEY WORDS. Cochliomyia macellaria, bowlfy, rearing methods, reprodutive behavior, secondary screwworm
\end{abstract}

As observações sobre os hábitos alimentares dos adultos de Cochliomyia macellaria (Fabricius, 1775) que incluem a necrofagia, a ingestão de exudados corporais, e resíduos alimentares urbanos (LAAKE et al. 1936; FERREIRA 1978, 1983; GUIMARÃES et al. 1983), correlacionados a surtos de algumas doenças infecto-contagiosas, sugerem a possibilidade da participação desta espécie na transmissão de patógenos, seja mecanicamente ou por regurgitação e excreção de material infectante (BISHOP 1915; GREENBERG 1971).

Os adultos de C. macellaria também se alimentam do néctar de flores, sendo, inclusive, atraídos por vegetais que emitem odores semelhantes à carne putrefata, tais como Aristolochia sp. e Iris foetidissima Linnaeus (GREENBERG 1971; TOMPKINS \& BIRD 1988). Entretanto, as informações relativas à biologia do adulto desta espécie são incipientes, existindo apenas algumas observações sobre o assunto (Bishop 1917; DeONier 1940; Oliveira et al. 1982; BaUmGartner \& GreENBERG 1985).

1) Departamento de Estudos Básicos e Instrumentais, Campus de Itapetinga, Universidade Estadual do Sudoeste da Bahia. 45700-000 Itapetinga, Bahia, Brasil.

2) Departamento de Parasitologia Animal, Universidade Federal Rural do Rio de Janeiro. 23851-970 Seropédica, Rio de Janeiro, Brasil.

Revta bras. Zool. 13 (4): 883 - 889, 1996 
Neste estudo, objetivou-se verificar a longevidade e o potencial reprodutivo de adultos de $C$. macellaria pertencentes a duas gerações e agrupados em duas diferentes densidades, em função de dois horários de coleta.

\section{MATERIAL E MÉTODOS}

Os adultos de C. macellaria originaram-se de um estoque mantido em laboratório sem a introdução de indivíduos nativos (CUNHA-E-SILVA \& MILWARDDE-AZEVEDo 1992). O estudo foi realizado em duas etapas e conduzido em câmara climatizada regulada a $27^{\circ} \mathrm{C}$ de temperatura, $65 \pm 10 \%$ UR e 14 horas de fotofase. A fotofase iniciava-se às 06:00 horas. Utilizou-se quatro repetições por etapa experimental. Na primeira etapa, utilizou-se 40 casais pertencentes à terceira geração, por repetição; na segunda etapa, 20 casais pertencentes à quarta geração. Logo após a emergência, os espécimens foram transferidos para gaiolas de madeira revestidas com tela de náilon, apresentando 235 e $57 \mathrm{~cm}^{3}$, respectivamente. Como substrato de alimentação, forneceu-se solução de mel a $50 \%$, carne eqüina putrefata e água destilada, trocadas diariamente. Como estímulo para a realização de posturas, utilizou-se carne eqüina putrefata, renovada duas vezes ao dia (08:00 e 17:00 horas). Este substrato foi previamente conservado em refrigerador e aquecido em estufa regulada a $30^{\circ} \mathrm{C}$ de temperatura, durante o intervalo de 24 horas, antes de sua utilização. As massas de ovos foram coletadas, diariamente, às 08:00 e 17:00 horas, pesadas e, em seguida, transferidas para recipientes de vidro transparente ( $9,0 \times 5,5 \mathrm{~cm}$ de diâmetro) forrados com papel de filtro umedecido com $0,3 \mathrm{ml}$ de água destilada e tampados com filme transparente de PVC. O período de incubação foi monitorado em câmara climatizada regulada a $30^{\circ} \mathrm{C}$ de temperatura, $65 \pm 10 \%$ UR e 14 horas de fotofase. Considerando-se que o período médio de incubação de $C$. macellaria, nestas condições, é de 12 horas (CUNHA-E-SILVA \& MILWARD-DE-AZEVEDO 1992), 24 horas após a coleta das massas de ovos este material foi fixado em álcool a $70 \%$ e, posteriormente, quantificado.

As curvas de sobrevivência, para machos e fêmeas, foram representadas através do modelo de distribuição proposto por Weibull e descrito por SGRILLO (1982).

Tabela I. Potencial biótico de fêmeas de Cochliomyia macellaria pertencentes à terceira ( 40 casais $\left./ 235 \mathrm{~cm}^{3}\right)$ e à quarta geração $\left(20\right.$ casais $\left./ 57 \mathrm{~cm}^{3}\right)$, em função de dois horários de coleta de massas de ovos. sob condicões controladas.

\begin{tabular}{lcccc}
\hline Geração & $\begin{array}{c}\text { Horário de } \\
\text { coleta } \\
(\mathrm{h})\end{array}$ & $\begin{array}{c}\text { Peso médio de } \\
\text { massa de ovos } \\
(\mathrm{g})\end{array}$ & $\begin{array}{c}\text { Número médio } \\
\text { de ovos }\end{array}$ & $\begin{array}{c}\text { Eclosão ** } \\
(\%)\end{array}$ \\
\hline Terceira & $08: 00$ & 0,15 & 1301,7 & 78,0 \\
& $17: 00$ & 1,53 & 12057,5 & 91,6 \\
Quarta & $08: 00$ & 0,52 & 4652,5 & 96,4 \\
& $17: 00$ & 0,59 & 4911,2 & 97,1 \\
\hline
\end{tabular}

${ }^{*} .27^{\circ} \mathrm{C}$ de temperatura, $65 \pm 10 \%$ UR, $14 \mathrm{~h}$ de fotofase; ${ }^{* *} .30^{\circ} \mathrm{C}$ de temperatura, $65 \pm 10 \%$ UR, $14 \mathrm{~h}$ de fotofase. 


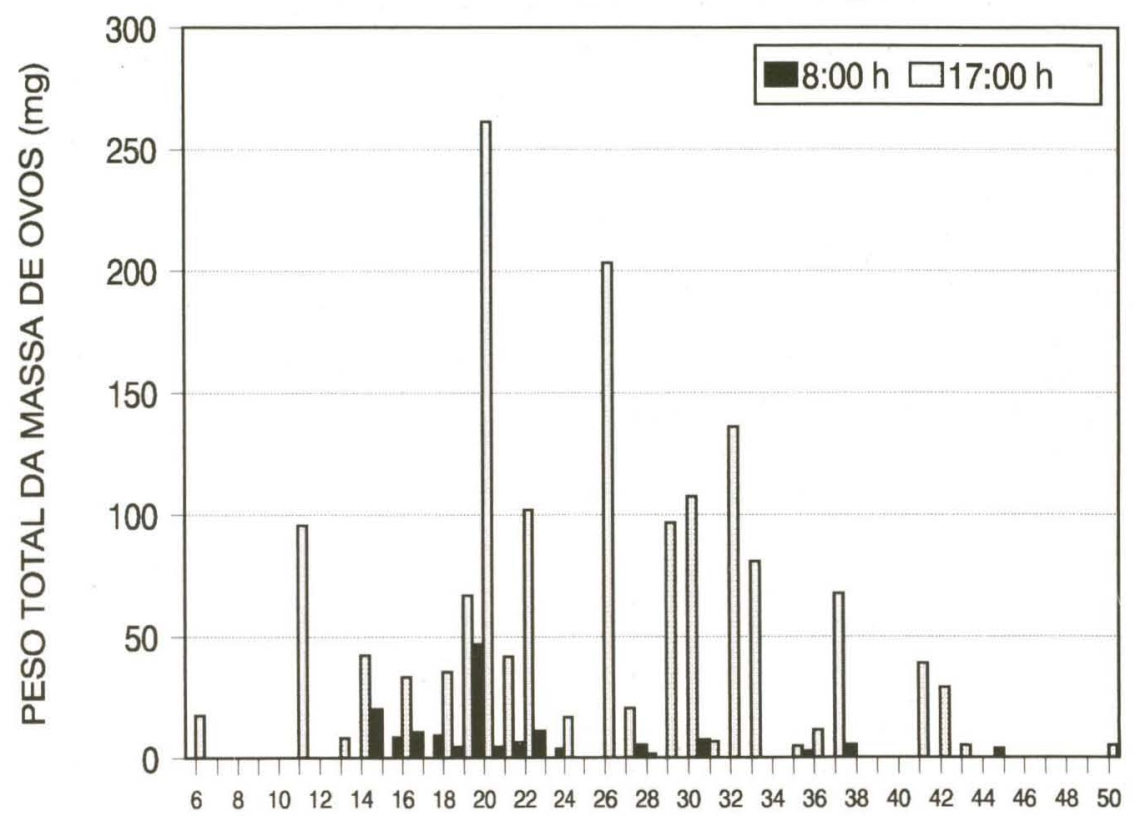

DIAS

Fig. 1. Ritmo de oviposição de Cochliomyia macellaria pertencente à terceira geração, utilizando-se a densidade inicial de 40 casais $/ 235 \mathrm{~cm}^{3}$ e em função de dois horários de coleta (temperatura $27^{\circ} \mathrm{C}, 65 \pm 10 \%$ UR e $14 \mathrm{~h}$ de fotofase).

\section{RESULTADOS E DISCUSSÃO}

O peso total de massa de ovos de C. macellaria, na primeira etapa experimental, foi significativamente maior na coleta realizada às 17:00 horas. $O$ horário de coleta não influenciou esta variável acentuadamente, durante a segunda etapa (Tab. I). Proporcionalmente, o potencial biótico estimado para os adultos da quarta geração mostrou-se superior ao registrado para a geração anterior, considerando-se a densidade de casais amostrados: 20 e 40 casais por parcela produziram, em média, o total de 1,11 e $1,68 \mathrm{~g}$ de ovos, respectivamente. Estes resultados refletiram-se no número total de ovos obtidos (Tab. I). As fêmeas pertencentes à terceira geração ovipositaram não somente sobre a carne putrefata, mas, eventualmente, sobre a superfície da tela que servia como apoio para o pouso durante a ingestão da solução de mel, em frestas, e na tela das gaiolas. Todas as massas de ovos obtidas fora do substrato à base de carne putrefata foram coletadas às 08:00 horas; nestas amostras, relativas à primeira etapa experimental, obteve-se uma maior percentagem de ovos inviáveis.

Na primeira e segunda etapa experimental, o pico de oviposição nas coletas realizadas às 8:00 horas, ocorreu no $20^{\circ} \mathrm{e} 17^{\circ}$ dia, respectivamente; e, às 17:00 horas, no $20^{\circ}$ e $11^{\circ}$ dia após a emergência das fềmeas (Figs 1,2). Comparativamente, 
registrou-se, portanto, um atraso no pico de oviposição no grupo relativo a 40 casais/gaiola.

As flutuações no comportamento reprodutivo desta espécie podem ser devidas ao grau de adaptação às condições de criação em cativeiro. Por outro lado, o efeito da densidade de casais $/ \mathrm{cm}^{3}$ de gaiola pode ter influenciado este comportamento (PETERS \& BARBOSA 1977). O efeito de grupo está intrinsecamente correlacionado às exigências metabólicas (que inclui o calor metabólico comportamental e/ou físiológico liberado) e à resposta dos indivíduos aos semioquímicos liberados. Assim, fatores interagentes (bióticos e abióticos) podem ter influenciado os resultados obtidos (SINGH \& MOORE 1985).

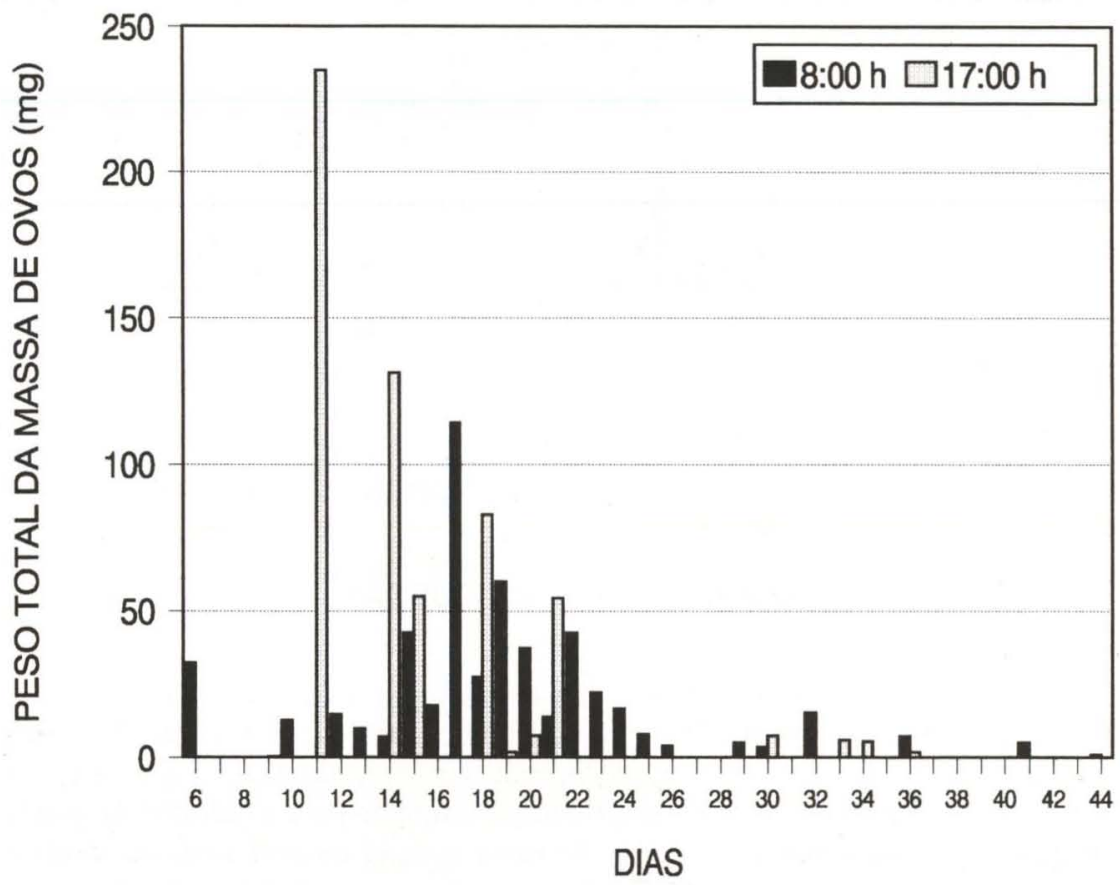

Fig. 2. Ritmo de oviposição de Cochliomyia macellaria pertencente à quarta geração, utilizando-se a densidade inicial de 20 casais $/ 57 \mathrm{~cm}^{3}$ e em função de dois horários de coleta (temperatura $27^{\circ} \mathrm{C}, 65 \pm 10 \%$ UR e $14 \mathrm{~h}$ de fotofase).

Machos e fêmeas de C. macellaria mostraram-se receptivos aos substratos alimentares (solução de mel e carne putrefata) oferecidos. BARTON-BROWNE et al. (1976) sugeriram que o acasalamento de espécimens selvagens de Phaenicia cuprina (Wiedemann, 1819) ocorre próximo a uma fonte proteica, quando as fêmeas estão obtendo alimento adicional. Estas evidências foram favorecidas pela captura de um número significativo de machos atraídos para as armadilhas com iscas (NORRIS 1966) e estimulados sexualmente pelo odor da carne putrefata (SHOREY et al. 1969). Fêmeas adultas de Calliphora vicina (Robineau-Desvoidy, 1830) 


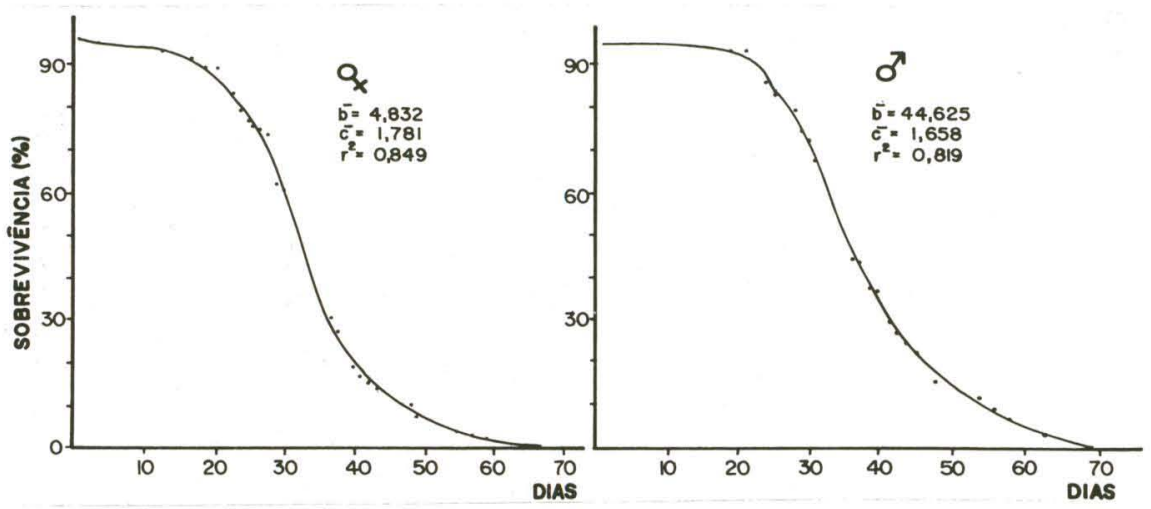

Fig. 3. Curvas de sobrevivência de machos e fêmeas de Cochliomyia macellaria pertencentes à terceira geração, apresentando a densidade inicial de 40 casais $/ 235 \mathrm{~cm}^{3}$ (temperatura $27^{\circ} \mathrm{C}$, $65 \pm 10 \%$ UR e $14 \mathrm{~h}$ de fotofase). Observado (•); esperado (-).

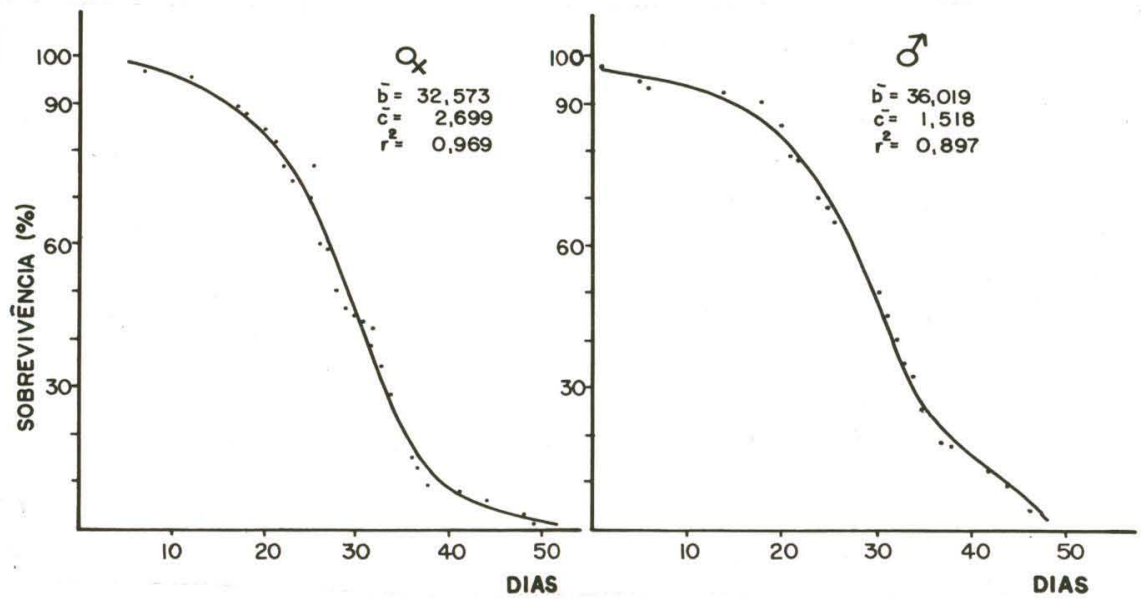

Fig. 4. Curvas de sobrevivência de machos e fêmeas de Cochliomyia macellaria pertencentes à quarta geração, apresentando a densidade inicial de 20 casais $/ 57 \mathrm{~cm}^{3}$ (temperatura $27^{\circ} \mathrm{C}$, $65 \pm 10 \%$ UR e $14 \mathrm{~h}$ de fotofase). Observado (•); esperado (-).

(STRAG- WAY-DiXon 1961) e Phormia regina (Meigen, 1830) (DETHIER \& Evans 1961) apresentaram flutuações cíclicas relativas à preferência alimentar quando foram oferecidas fonte proteiça animal e solução aquosa de açúcar, em testes de livre escolha. Os autores correlacionaram estas flutuações com a periodicidade no crescimento ovariano. Por outro lado, no presente trabalho, durante as observações diárias, C. macellaria não se mostrou receptiva ao fornecimento de água pura, demonstrando preferência pela solução de mel a 50\%. BARTON-BROWNE (1962) verificou que o contato labelar de $P$. cuprina com a água não foi significativo $\mathrm{e}$, portanto, ela não foi exigida como complemento dietético, por esta espécie. Nossas observações sugeriram que, provavelmente, em condições experimentais, C. mace- 
llaria também não necessite do fornecimento adicional de água, visto que a mesma foi ingerida através da sua associação com o mel.

Os adultos pertencentes à terceira geração apresentaram-se, em média, apenas alguns dias mais longevos do que as imagos da progênie subsequente: $50 \%$ dos indivíduos sobreviveram de 30 a 35 dias (Figs 3, 4). Esta variável, portanto, não explicou as diferenças entre as tendências sugeridas pelos ritmos de oviposição apresentadas pelas duas amostras e discutidos anteriormente.

\section{REFERÊNCIAS BIBLIOGRÁFICAS}

BARTON-BROWNE, L.B. 1962. The relationship between oviposition in the blowfly Lucilia cuprina and the presence of water. Insect Physiol. 8: 383-390.

BARTON-BRowne, L.; R.J. BARTELL; A.C.M. VANGERWEN \& L.A. LAWRENCE. 1976. Relationship between protein ingestion and sexual receptivity in females of the Australian sheep blowfly Lucilia cuprina. Physiol. Entomol. 1: 235-240. BAumgartneR, D.L. \& B. GREENBERG. 1985. Distribution and medical ecology of the blowflies (Diptera: Calliphoridae) of Peru. Ann. ent. Soc. Amer. 78: 565-587.

BISHOP, F.C. 1915. Flies wich cause myiasis in man and animals some aspects of the problem. Jour. Econ. Entomol. 8: 317-329.

- 1917. Some problems in insect control and packing houses. Jour. Econ. Entomol. 10: 269-277.

CunHA-E-SiLva, S.L. \& E.M.V. MiLwARD-DE-AZEvedo. 1992. Estudo comparado do desenvolvimento de dois morfotipos larvas de Cochliomyia macellaria (Fabricius) (Diptera: Calliphoridae). Revta bras. Zool. 9: 181-186.

DEONIER, C.C. 1940. Carcass temperatures and their relation to winter blowfly populations and activity in the south west. Jour. Econ. Entomol. 3: 166-170.

DethiER, V.G. \& D.T. Evans. 1961. The physiological control of water ingestion in the blowfly. Biol. Bull. 121: 108-116.

FERreIRA, M.J.M. 1978. Sinantropia de dípteros muscoideos de Curitiba, Paraná.

I. Calliphoridae. Rev. Brasil. Biol. 38: 445-454.

_ 1983. Sinantropia de Calliphoridae (Diptera) em Goiânia, Goiás. Rev. Brasil. Biol. 43: 199-210.

GREENBERG, B. 1971. Flies and Disease, vol. I: Ecology, classification and biotic associations. New York, Princeton Univ. Press, 865p.

Guimarães, J.H.; N. Papavero \& A.P. Prado. 1983. As míases na região neotropical. Revta bras. Zool. 1 (4): 239-416.

LAAKE, E.W.; E.C. GUSHING \& H.E. PARISH. 1936. Biology of the primary screwworm fly, Cochliomyia americana, and a comparison of it's stages with those of C. macellaria. U.S. Dep. Agric. Tech. Bull. 500: 1-24.

NORRIS, K.R. 1966. Daily patterns of flightactivity of blowflies (Calliphoridae: Diptera) in the Camberra district as indicated by trap catches. Aust. Jour. Zool. 14: 835-853.

OliveirA, C.M.B.; G.E. MoyA \& R.P. MelLo. 1982. Flutuação populacional de 
Cochliomyia hominivorax no Município de Itaguaí, Rio de Janeiro. Pesq. Vet. Brasil. 2 (4): 139-142.

PETERS, T.M. \& P. BARBosA. 1977. Influence of population density on size, fecundity, and developmental rate of insects in culture. Ann. Rev. Entomol. 22: 431-450.

SGRILlo, R.B. 1982. A distribuição de Weibull como modelo de sobrevivência de insetos. Ecossistema 7: 9-13.

Shorey, H.H.; R.J. BARTELL \& L.B. BARTON BRowne. 1969. Sexual stimulation of males of Lucilia cuprina (Calliphoridae) and Drosophila melanogaster (Drosophilidae) by the odors of aggregation sites. Ann. ent. Soc. Amer. 62: 1419-1421.

SINGH, P. \& R.F. MOORE. 1985. Handbook of Insect Rearing. Elsevier, Vol.1, VIII+488p.

STRANGWAY-DiXON, J. 1961. The relationship between nutritions hormones and reproduction in the blowfly Calliphora erythrocephala (Meigen). I. Selective feeding in relation to the reproductive cycle, the corpus allatum, volume and fertilization. I. Exp. Biol. 38: 225-235.

TOMPKINS, P. \& C. BIRD. 1988. A vida secreta das plantas. Expressão e Cultura, $324 \mathrm{p}$.

Recebido em 20.VIII.1994; aceito em 28.XII.1996. 